\title{
Clinical effect of immunophenotyping on the prognosis of multiple myeloma patients treated with bortezomib
}

\author{
NORIYOSHI IRIYAMA ${ }^{1}$, KATSUHIRO MIURA ${ }^{1}$, YOSHIHIRO HATTA ${ }^{1}$, SUMIKO KOBAYASHI $^{1}$, \\ YOSHIHITO UCHINO $^{1}$, DAISUKE KURITA ${ }^{1}$, HITOMI SAKAGAMI $^{1}$, HIROMICHI TAKAHASHI ${ }^{1,2}$, \\ MASASHI SAKAGAMI ${ }^{1}$, YUJIN KOBAYASHI ${ }^{1}$, MASARU NAKAGAWA ${ }^{1}$, SHIMON OHTAKE ${ }^{1}$, \\ YOSHIKAZU IIZUKA $^{1}$ and MASAMI TAKEI ${ }^{1}$ \\ ${ }^{1}$ Division of Hematology and Rheumatology, Department of Medicine; ${ }^{2}$ Department of Clinical Laboratory, \\ Nihon University School of Medicine, Tokyo 173-8610, Japan
}

Received March 28, 2016; Accepted January 19, 2017

DOI: $10.3892 / \mathrm{ol} .2017 .5920$

\begin{abstract}
In the present study, the effect of immunophenotyping on the prognoses of patients with multiple myeloma (MM) treated with bortezomib plus dexamethasone was investigated. The study involved 46 patients with MM, and analyzed the prognostic significance of the expression of cluster of differentiation (CD)45, CD56 and mature plasma cell (MPC)-1, and other factors including the International Staging System (ISS) stage, age, gender, the immunoglobulin subtype and the treatment line number prior to bortezomib treatment. Although CD56 and MPC-1 expression did not appear to affect the time to next treatment (TNT) or overall survival rate (OS), the univariate analysis determined that CD45 positivity was an adverse prognostic factor for TNT and OS, and that being male was significantly associated with inferior TNT and OS. Multivariate analyses determined that CD45 expression was prognostically significant for TNT and OS. In conclusion, CD45 positivity is an adverse prognostic factor in MM patients treated with bortezomib. The data from the present study demonstrate the clinical importance of classifying MM cells immunophenotypically to determine the prognoses of patients.
\end{abstract}

\section{Introduction}

Multiple myeloma (MM) is a hematologic malignancy that is characterized by an increased number of abnormal plasma cells in the bone marrow. Whilst curing MM is difficult, the

Correspondence to: Dr Noriyoshi Iriyama or Dr Yoshihiro Hatta, Division of Hematology and Rheumatology, Department of Medicine, Nihon University School of Medicine, 30-1 Oyaguchi Kami-cho, Itabashi-ku, Tokyo 173-8610, Japan

E-mail: iriyama.noriyoshi@nihon-u.ac.jp

E-mail: hatta.yoshihiro@nihon-u.ac.jp

Key words: multiple myeloma, bortezomib, cluster of differentiation 45 , cluster of differentiation 56, mature plasma cell-1 recent development of novel agents including bortezomib and lenalidomide has led to considerable improvements in the outcomes of patients with MM (1-3). The main approved treatment strategy prior to bortezomib was to use chemotherapeutic agents with or without autologous stem cell transplantation (ASCT). Bortezomib became available in 2006 in Japan, and patients with MM who had relapsed or were refractory to chemotherapy were subsequently treated with bortezomib-based regimens. Furthermore, as bortezomib is available as a first-line treatment at present for incident diagnoses of MM, the treatment approach for MM has changed and bortezomib is used as an initial treatment.

It is important to be able to predict patient outcomes in clinical practice. Although the presence of specific cytogenetic abnormalities within myeloma cells, such as $\mathrm{t}(4 ; 14)$ and $\mathrm{t}(11 ; 14)$, is considered the most important predictor of the prognoses of patients who have been treated with chemotherapeutic agents, the data from several previous studies have demonstrated that bortezomib-based treatment regimens may be able to overcome the adverse prognostic effects of these abnormalities (4-7). The International Staging System (ISS), which is a widely used prognostic indicator, may also have little effect on patient outcome predictions in this era of novel therapeutic agents (8-10). Thus, the prognostic factors in patients with MM who are treated using bortezomib-based regimens remain unclear.

Immunophenotyping is widely available for the diagnosis of, the evaluation of minimal residual disease in and the prediction of the prognoses of patients in terms of a variety of hematologic malignancies, including MM. Myeloma cells commonly show the cluster of differentiation (CD) $38^{\text {bright }} \mathrm{CD} 19^{-} \mathrm{CD} 56^{+}$immunophenotype and, occasionally, the CD38 ${ }^{\text {bright }} \mathrm{CD} 19^{-} \mathrm{CD} 56^{-}$immunophenotype, which may be clearly distinguished from the immunophenotype shown by normal plasma cells, CD38 ${ }^{\text {bright }} \mathrm{CD} 19^{+} \mathrm{CD} 56^{-}(11,12)$. Furthermore, unlike normal plasma cells, myeloma cells often do not express the mature plasma cell (MPC)-1 antigen or the leukocyte common antigen (CD45) (13-15). The prognostic interpretation of this distinct immunophenotypic pattern in MM has been studied previously. CD56 negativity in myeloma cells is considered to be an adverse prognostic factor (16-18), 
and patients with MM whose cells express CD45 survive longer compared with those patients whose cells are negative for CD45, particularly patients who have been treated with ASCT $(14,19)$. A reduction in MPC-1 expression may be associated with chemotherapy or thalidomide resistance (13). However, all of the previous studies that have examined the effect of immunophenotyping on prognosis involved patients who had been treated with chemotherapy with or without ASCT. Therefore, there are few data concerning the clinical significance of immunophenotyping for patients with MM who have been treated with novel agents such as bortezomib. Thus, a comprehensive analysis of the significance of immunophenotyping for patients with MM who have been treated with bortezomib is required. Therefore, the present study analyzed data from patients with MM who had been treated with bortezomib at Nihon University School of Medicine (Tokyo, Japan). The present study describes the significance of immunophenotyping for patients treated with bortezomib, and discusses the molecular diversity that may underlie the pathogenesis of MM in the context of immunophenotypic classification.

\section{Patients and methods}

Patients and treatment. A retrospective review of data from patients between December 2006 and August 2015 was conducted. The present study included symptomatic patients with MM who were treated with bortezomib plus dexamethasone as a second-line therapy or subsequently, and excluded those who received cytotoxic agents, including melphalan or cyclophosphamide, in combination with bortezomib. Baseline characteristics of patients at the time of bortezomib initiation, including age, gender, immunoglobulin subtype, ISS (8-10), pretreatments and immunophenotypes on myeloma cells, were investigated for the assessment of prognostic significance. Bortezomib was administered either intravenously or subcutaneously at a dose of $1.3 \mathrm{mg} / \mathrm{m}^{2}$ on days $1,4,8$ and 11 , every 3 weeks, or on days $1,8,15$ and 22 , every 5 weeks, in combination with oral dexamethasone that was administered at a maximum dose (40 mg/day) on days 1-4, 9-12 and 17-20 or at a reduced dose dependent on patient background, and it was administered intravenously (between December 2006 and December 2012) or subcutaneously (between January 2013 and August 2015). The study was approved by the research ethics board of Nihon University Itabashi Hospital (Identifier: RK-151208-06, approved in January 2016), and the study was conducted in accordance with the principles of the Declaration of Helsinki.

Flow cytometry. The immunophenotyping was performed primarily on bone marrow samples that were collected at the time of diagnosis or prior to bortezomib treatment at Bio Medical Laboratories, Inc. (Tokyo, Japan). The myeloma cells were analyzed using standard immunofluorescence methods and monoclonal antibodies. Briefly, nuclear cells isolated from patient bone marrow samples were washed with PBS and stained with fluorescein isothiocyanate-labeled mouse anti-CD38 (1:20; cat. no. 555459; Becton-Dickinson, San Jose, CA, USA), phycoerythrin (PE)-labeled mouse anti-CD56 (1:20; cat. no. 347747; Becton-Dickinson), PE-labeled
Table I. Baseline characteristics of patients at the time of bortezomib initiation.

\begin{tabular}{lc}
\hline Characteristic & $\mathrm{n}=46$ \\
\hline Age, years, median (range) & $65(36-83)$ \\
Gender, $\mathrm{n}$, male/female & $27 / 19$ \\
Immunoglobulin subtype (n) & \\
IgG & 30 \\
IgA & 6 \\
IgD & 1 \\
Light chain only & 7 \\
Non-secretory & 2 \\
International Staging System, $\mathrm{n}$ & \\
Stage I & 16 \\
Stage II & 10 \\
Stage III & 15 \\
Missing & 5 \\
Pretreatment, $\mathrm{n}$ & \\
MP & 32 \\
Multi-agent chemotherapy & 17 \\
Thalidomide & 8 \\
High-dose dexamethasone & 6 \\
aPBSCT & 5 \\
Lenalidomide & 3 \\
CP & 1 \\
Pretreatment line number, $\mathrm{n}$ & \\
1 & 29 \\
2 & 14 \\
$\geq 3$ & 3 \\
\hline
\end{tabular}

Ig, immunoglobulin; MP, melphalan plus prednisolone; aPBSCT, autologous peripheral blood cell transplantation; $\mathrm{CP}$ cyclophosphamide and prednisolone.

mouse anti-MPC-1 (1:10; cat. no. 03524781-4; Otsuka, Tokyo, Japan), PE-labeled mouse anti-CD49e (1:80; cat. no. 555617; Becton-Dickinson), peridinin-chlorophyll-protein complex-labeled mouse anti-CD45 (1:20; cat. no. 347764; Becton-Dickinson), or allophycocyanin-labeled mouse anti-CD19 (1:40; cat. no. IM2470; Beckman Coulter, Brea, CA, USA) for $30 \mathrm{~min}$ at $4^{\circ} \mathrm{C}$ in the dark. Cells were then washed with PBS and analyzed by flow cytometry with a minimum acquisition of 20,000 events. Double-gating analyses were performed with CD38/side scatter, CD19*CD56, CD45*MPC-1 and $\mathrm{CD} 45 * \mathrm{CD} 49 \mathrm{e}$. Values were calculated using CELLQuest, version 3.3 (Becton-Dickinson) and analyzed using original software from BML. Non-binding mouse isotype antibodies were used as controls. The CD $38^{\text {bright }} /$ side scatter low $^{\text {population }}$ represented the plasma cell fraction, and the flow cytometric data were analyzed when abnormal plasma cell fractions, for example, CD $38^{\text {bright }} / \mathrm{CD} 19^{-}$cells, were detected. The samples were considered positive when at least $20 \%$ of the myeloma cells expressed this antigen profile, as described previously (20-22). 

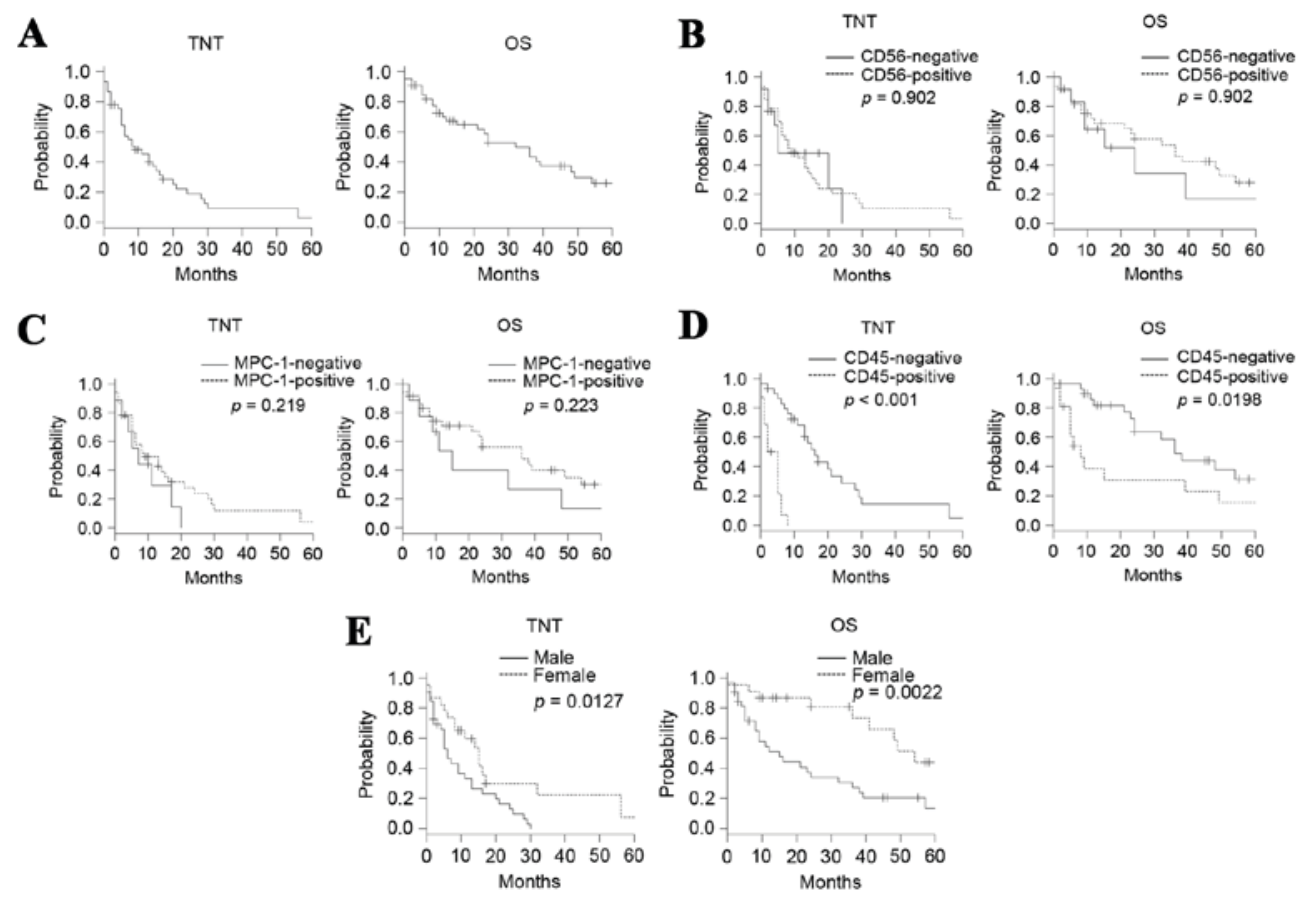

Figure 1. Kaplan-Meier curves for TNT and OS in (A) the entire cohort according to the immunophenotyping profile, including (B) CD56, (C) MPC-1, (D) CD45 and (E) patient gender. Neither the CD56 nor the MPC-1 status exhibited any effect on TNT or OS. CD45 positivity and male gender were adverse prognostic factors that affected TNT and OS. TNT, time to next treatment; OS, overall survival rate; CD, cluster of differentiation; MPC-1, mature plasma cell-1.

Statistical analysis. Time to next treatment (TNT) was defined as the period from the date that bortezomib treatment was initiated to the date where subsequent therapy other than bortezomib plus dexamethasone began or the patient succumbed to any cause. The overall survival rate (OS) was defined as the period from the date that bortezomib treatment was initiated to the date of death. The Kaplan-Meier method was used to estimate the TNT and OS, and the log-rank test was used to compare the groups in association with the TNT and OS. The factors that may affect the clinical outcomes were analyzed using multivariate Cox proportional hazard regression models. $\mathrm{P}<0.05$ was considered to indicate a statistically significant difference. The statistical analyses were performed using Easy R (Saitama Medical Center, Jichi Medical University), which is a graphical user interface for the $\mathrm{R}$ programming language (The $\mathrm{R}$ Foundation for Statistical Computing, Vienna, Austria; http://www.R-project.org/) (23).

\section{Results}

Characteristics and treatment of patients. Of the patients diagnosed with symptomatic MM, 56 had been treated with bortezomib plus dexamethasone between December 2006 and August 2015. Of these 56 patients, 46 were available for the immunophenotyping assessment. The characteristics of these enrolled patients are presented in Table I. The median age at diagnosis was 65 years, range 36-83 years, and the present study included 27 men and 19 women. Following the ISS assessments, 16, 10 and 15 patients were classified as being at stages I, II and III, respectively, at the time bortezomib treatment was initiated.
Immunophenotyping and prognosis. The flow cytometric data attained prior to bortezomib treatment were available for 46 patients. Of the patients evaluated, CD45, CD49e, CD56 and MPC-1 were positively expressed in 16 (35\%), 2 (4\%), 33 (72\%) and $37(80 \%)$ patients, respectively. Due to the low number of patients who possessed myeloma cells that expressed CD49e, the present study focused on the expression of CD45, CD56 and MPC-1 during the analysis of the flow cytometric data.

The median TNT and the median survival time (MST) were 9 and 32 months, respectively, for the entire cohort (Fig. 1A). The TNT and OS did not differ significantly between the patients who were positive and those who were negative for CD56, with median TNTs of 9 and 5 months, respectively, and MSTs of 36 and 24 months, respectively (Fig. 1B). The TNTs and OS did not differ significantly between the patients who were positive and those who were negative for MPC-1, with median TNTs of 9 and 7 months, respectively, and MSTs of 36 and 15 months, respectively (Fig. 1C). Patients who were positive for CD45 expression exhibited shorter TNTs and OS compared with those who were negative for CD45 expression, and the median TNTs were 3.5 and 16 months, respectively, and MSTs were 8 and 36 months, respectively (Fig. 1D). Thus, among the surface antigens evaluated, CD45 positivity was the only factor that affected the outcomes of the patients.

Prognostic factors other than surface antigen expression. Following the ISS assessments at the initiation of bortezomib treatment, the median TNTs for the patients at stages I, II or III were 11,8 and 11 months, respectively $(\mathrm{P}=0.45)$, and the $\mathrm{OS}$ for the patients at ISS stages I, II or III were 36, 49 and 24 months, respectively $(\mathrm{P}=0.084)$. Patient age, the pretreatment line 
Table II. Analysis of the risk factors associated with the time to next treatment in the study population $(n=46)$.

\begin{tabular}{|c|c|c|c|c|}
\hline \multirow[b]{2}{*}{ Factor } & \multicolumn{2}{|c|}{ Univariate analysis } & \multicolumn{2}{|c|}{ Multivariate analysis } \\
\hline & HR $(95 \% \mathrm{CI})$ & P-value & HR (95\% CI) & P-value \\
\hline Male sex & $2.22(1.08-4.55)$ & 0.030 & $1.89(0.90-3.95)$ & 0.092 \\
\hline CD45 positivity & $9.80(3.71-25.9)$ & $<0.0001$ & $9.41(3.44-25.7)$ & $<0.0001$ \\
\hline
\end{tabular}

HR, hazard ratio; $\mathrm{CI}$, confidence interval; $\mathrm{CD}$, cluster of differentiation.

Table III. Analysis of the risk factors for overall survival in the study population $(n=46)$.

\begin{tabular}{|c|c|c|c|c|}
\hline \multirow[b]{2}{*}{ Factor } & \multicolumn{2}{|c|}{ Univariate analysis } & \multicolumn{2}{|c|}{ Multivariate analysis } \\
\hline & HR (95\% CI) & P-value & HR (95\% CI) & P-value \\
\hline Male sex & $2.35(1.02-5.42)$ & 0.044 & $2.15(0.93-5.00)$ & 0.074 \\
\hline CD45 positivity & $2.40(1.12-5.18)$ & 0.025 & $2.19(1.01-4.77)$ & 0.047 \\
\hline
\end{tabular}

HR, hazard ratio; $\mathrm{CI}$, confidence interval; $\mathrm{CD}$, cluster of differentiation.

number and the immunoglobulin subtype did not affect the TNT or OS. However, being male adversely affected the TNT and OS (Fig. 1E).

Univariate and multivariate analyses. The univariate analysis revealed that compared with the female patients, the TNT was significantly shorter for the male patients and it also determined that CD45 positivity was associated with a shorter TNT. The multivariate analysis revealed that CD45 positivity was an independent adverse prognostic factor for the TNT (Table II) and OS (Table III). The multivariate analysis demonstrated that being male was not an adverse prognostic factor for TNT (Table II) and OS (Table III).

\section{Discussion}

The data from the present study demonstrate the prognostic significance of the immunophenotype for patients with MM who are treated with bortezomib, and they differ from the data of previous studies of patients who underwent treatment prior to the introduction of novel therapeutic agents (14-19). Notably, the OS data from the present study may have been affected by treatment that was administered subsequent to bortezomib such as lenalidomide. Indeed, 25 patients in the present study received lenalidomide and 7 patients received thalidomide as salvage therapy subsequent to bortezomib administration. However, none of the patients underwent ASCT.

Notably, data from the present study demonstrated that the expression of CD45 by myeloma cells was a critical adverse prognostic factor that affected the TNT and OS, which conflicts with previously published data that suggested favorable outcomes for patients with MM who were CD45 positive and were treated with chemotherapy and ASCT, but were not administered bortezomib $(14,19)$. The levels of
CD45 expression are increased subsequent to bortezomib treatment (24), which suggests an association between the CD45 molecule and bortezomib resistance. Therefore, the present study hypothesized that the contradictory nature of these data with respect to the findings of previous studies is important for understanding the critical role of a treatment strategy that involves combination therapy, for example, bortezomib-based treatment followed by ASCT for patients with MM, and that it may assist in explaining the different drug resistance mechanisms that occur between bortezomib and chemotherapeutic agents. Differences in the characteristics of myeloma cells in terms of the expression of CD45 have been studied comprehensively. The CD45-positive fraction of myeloma cells is a growth component that responds to interleukin-6 (IL-6) (25-27), which is a critical factor that regulates cell growth and survival through the autocrine or paracrine systems of the myeloma cells. In addition, IL-6 serves a major role in the activation of the Janus family tyrosine kinase-signal transducer and activator of transcription (JAK-STAT) signaling pathway, mainly via signal STAT3 and STAT 5 (28). Collectively, these data suggest that the CD45-positive component of myeloma cells exhibits a high level of activity that is associated with the JAK-STAT pathway, and that the activation of the CD45 component may be associated with poor responses to bortezomib. The findings from a recent study that used phospho-flow cytometry demonstrated that CD45-positive myeloma cells were associated with higher levels of STAT3 and/or STAT5 activity (29). Furthermore, patients with MM who exhibited higher levels of STAT3 and STAT5 phosphorylation exhibited favorable outcomes when they were treated with chemotherapy with or without ASCT (29). These results support the hypothesis that the critical pathways for the pathogenesis and/or development of MM differ according to the CD45 status of the patient. Therefore, it may be suggested that the outcomes of patients 
treated with bortezomib may depend on the underlying mechanisms that promote myeloma development in each patient.

ASCT is considered to be a valid treatment option for younger patients with MM. The data of the present study demonstrated that patients with MM who were CD45 positive exhibited poorer outcomes. When the different prognostic interpretations of CD45 expression are considered in association with the treatment strategy, ASCT may compensate for bortezomib treatment. Therefore, the present study proposed that patients with MM who are administered bortezomib-based treatment should undergo ASCT, particularly when the myeloma cells are positive for CD45 and the patient is able to tolerate ASCT.

CD56 expression is common in myeloma cells, but not in normal plasma cells. A lack of CD56 expression has been demonstrated to be associated with poorer outcomes when ASCT is not undertaken, but the negative effect of an absence of CD56 expression is overcome when patients undergo ASCT $(16-18,30)$. Although the present study did not investigate the effect of ASCT on OS, the expression of CD56 did not affect OS, which suggests that the bortezomib-based regimen may overcome the prognostic significance of CD56 negativity. Additionally, the maturity of the myeloma cells, which was indicated by the expression of MPC-1, was not a predictor of the TNT or $\mathrm{OS}$ in the present study. However, a reduction in the expression of MPC-1 in some patients was identified, including those who were administered bortezomib as first-line therapy, who were refractory to or had relapsed subsequent to bortezomib treatment, suggesting that MPC-1-negative clones may be associated with bortezomib resistance. Additional studies are required to clarify the association between MPC-1 expression and the treatment outcomes.

In the present study, cytogenetic analyses using fluorescence in situ hybridization to detect specific cytogenetic abnormalities were not performed in the majority of cases, which is a limitation of the present study. Another limitation is associated with possible selection bias, as not all of the samples of the patients underwent flow cytometric analysis at the time of diagnosis or prior to bortezomib treatment. Furthermore, the treatment strategy was not uniform at Nihon University School of Medicine, due to the comorbidities of the patients and/or the discretion of the physicians.

In conclusion, patients with MM may be stratified according to their immunophenotypic classifications to predict outcomes, and this may assist to plan treatment. Consecutive evaluations of surface antigen expression by myeloma cells may serve a role in treatment optimization.

\section{Acknowledgements}

Dr Katisuhiro Miura and Dr Yohihiro Hatta received lecture fees and honoraria from Celgene K.K. and Janssen Pharmaceutical K.K. Professor Masami Takei received honoraria from Janssen Pharmaceutical K.K.

\section{References}

1. Richardson PG, Barlogie B, Berenson J, Singhal S, Jagannath S Irwin D, Rajkumar SV, Srkalovic G, Alsina M, Alexanian R, et al: A phase 2 study of bortezomib in relapsed, refractory myeloma. N Engl J Med 348: 2609-2617, 2003.
2. Richardson PG, Sonneveld P, Schuster MW, Irwin D, Stadtmauer EA, Facon T, Harousseau JL, Ben-Yehuda D, Lonial S, Goldschmidt $\mathrm{H}$, et al: Bortezomib or high-dose dexamethasone for relapsed multiple myeloma. N Engl J Med 352: 2487-2498, 2005.

3. Dimopoulos M, Spencer A, Attal M, Prince HM, Harousseau JL, Dmoszynska A, San Miguel J, Hellmann A, Facon T, Foà R, et al: Lenalidomide plus dexamethasone for relapsed or refractory multiple myeloma. N Engl J Med 357: 2123-2132, 2007.

4. Avet-Loiseau H, Leleu X, Roussel M, Moreau P, Guerin-Charbonnel C, Caillot D, Marit G, Benboubker L, Voillat L, Mathiot $\mathrm{C}$, et al: Bortezomib plus dexamethasone induction improves outcome of patients with $\mathrm{t}(4 ; 14)$ myeloma but not outcome of patients with del(17p). J Clin Oncol 28: 4630-4634, 2010.

5. Chang H, Trieu Y, Qi X, Xu W, Stewart KA and Reece D: Bortezomib therapy response is independent of cytogenetic abnormalities in relapsed/refractory multiple myeloma. Leuk Res 31: 779-782, 2007.

6. Jagannath S, Richardson PG, Sonneveld P, Schuster MW, Irwin D, Stadtmauer EA, Facon T, Harousseau JL, Cowan JM and Anderson KC: Bortezomib appears to overcome the poor prognosis conferred by chromosome 13 deletion in phase 2 and 3 trials. Leukemia 21: 151-157, 2007.

7. Sagaster V, Ludwig H, Kaufmann H, Odelga V, Zojer N, Ackermann J, Küenburg E, Wieser R, Zielinski C and Drach J: Bortezomib in relapsed multiple myeloma: Response rates and duration of response are independent of a chromosome 13q-deletion. Leukemia 21: 164-168, 2007.

8. Kuroda J, Shimura Y, Ohta K, Tanaka H, Shibayama H, Kosugi S, Fuchida S, Kobayashi M, Kaneko H and Uoshima N: Limited value of the international staging system for predicting long-term outcome of transplant-ineligible, newly diagnosed, symptomatic multiple myeloma in the era of novel agents. Int J Hematol 99: 441-449, 2014.

9. Tan D, Kim K, Kim JS, Eom HS, Teoh G, Ong KH, Goh YT, Durie BG, Chng WJ and Lee JH: The impact of upfront versus sequential use of bortezomib among patients with newly diagnosed multiple myeloma (MM): A joint analysis of the Singapore MM Study Group and the Korean MM Working Party for the Asian myeloma network. Leuk Res 37: 1070-1076, 2013.

10. Maltezas D, Dimopoulos MA, Katodritou I, Repousis P, Pouli A, Terpos E, Panayiotidis P, Delimpasi S, Michalis E and Anargyrou K: Re-evaluation of prognostic markers including staging, serum free light chains or their ratio and serum lactate dehydrogenase in multiple myeloma patients receiving novel agents. Hematol Oncol 31: 96-102, 2013.

11. Harada H, Kawano MM, Huang N, Harada Y, Iwato K, Tanabe O, Tanaka H, Sakai A, Asaoku H and Kuramoto A: Phenotypic difference of normal plasma cells from mature myeloma cells. Blood 81: 2658-2663, 1993.

12. Kawano MM, Mihara K, Tsujimoto T, Huang N and Kuramoto A: A new phenotypic classification of bone marrow plasmacytosis. Int J Hematol 61: 179-188, 1995.

13. Kuroda Y, Sakai A, Okikawa Y,Munemasa S, Katayama Y,HyodoH, Imagawa J, Takimoto Y, Okita H, Ohtaki $\mathrm{M}$ and Kimura A: The maturation of myeloma cells correlates with sensitivity to chemotherapeutic agents. Int J Hematol 81: 335-341, 2005.

14. Kumar S, Rajkumar SV, Kimlinger T, Greipp PR and Witzig TE: CD45 expression by bone marrow plasma cells in multiple myeloma: Clinical and biological correlations. Leukemia 19: 1466-1470, 2005.

15. Otsuyama K, Asaoku $\mathrm{H}$ and Kawano MM: An increase in MPC-1 and MPC-1-CD45 immature myeloma cells in the progressive states of bone marrow plasmacytosis: The revised phenotypic classification of monoclonal marrow plasmacytosis (MOMP-2005). Int J Hematol 83: 39-43, 2006.

16. Sahara N, Takeshita A, Shigeno K, Fujisawa S, Takeshita K, Naito K, Ihara M, Ono T, Tamashima S, Nara K, et al: Clinicopathological and prognostic characteristics of CD56-negative multiple myeloma. Br J Haematol 117: 882-885, 2002.

17. Sahara N and Takeshita A: Prognostic significance of surface markers expressed in multiple myeloma: CD56 and other antigens. Leuk Lymphoma 45: 61-65, 2004.

18. Chang H, Samiee S and Yi QL: Prognostic relevance of CD56 expression in multiple myeloma: A study including 107 cases treated with high-dose melphalan-based chemotherapy and autologous stem cell transplant. Leuk Lymphoma 47: 43-47, 2006.

19. Moreau P, Robillard N, Avet-Loiseau H, Pineau D, Morineau N, Milpied N, Harousseau JL and Bataille R: Patients with CD45 negative multiple myeloma receiving high-dose therapy have a shorter survival than those with CD 45 positive multiple myeloma. Haematologica 89: 547-551, 2004. 
20. Bene MC, Castoldi G, Knapp W, Ludwig WD, Matutes E, Orfao A and van't Veer MB: Proposals for the immunological classification of acute leukemias. European group for the immunological characterization of leukemias (EGIL). Leukemia 9: 1783-1786, 1995.

21. Iriyama N, Asou N, Miyazaki Y, Yamaguchi S, Sato S, Sakura T, Maeda T, Handa H, Takahashi M, Ohtake S, et al: Normal karyotype acute myeloid leukemia with the $\mathrm{CD} 7^{+} \mathrm{CD} 15^{+} \mathrm{CD} 34^{+} \mathrm{HLA}-\mathrm{DR}$ + immunophenotype is a clinically distinct entity with a favorable outcome. Ann Hematol 93: 957-563, 2014.

22. Iriyama N, Hatta Y, Takeuchi J, Ogawa Y, Ohtake S, Sakura T, Mitani K, Ishida F, Takahashi M, Maeda T, et al: CD56 expression is an independent prognostic factor for relapse in acute myeloid leukemia with $\mathrm{t}(8 ; 21)$. Leuk Res 37: 1021-1026, 2013.

23. Kanda Y: Investigation of the freely available easy-to-use software 'EZR' for medical statistics. Bone Marrow Transplant 48: 452-458, 2013.

24. Tagoug I, Plesa A and Dumontet C: Bortezomib influences the expression of malignant plasma cells membrane antigens. Eur J Pharmacol 706: 11-16, 2013

25. Mahmoud MS, Ishikawa H, Fujii R and Kawano MM: Induction of CD45 expression and proliferation in U-266 myeloma cell line by interleukin-6. Blood 92: 3887-3897, 1998.
26. Fujii R, Ishikawa H, Mahmoud MS, Asaoku $H$ and Kawano MM: MPC-1-CD49e- immature myeloma cells include CD $45^{+}$ subpopulations that can proliferate in response to IL-6 in human myelomas. Br J Haematol 105: 131-140, 1999.

27. Ishikawa H, Tsuyama N, Abroun S, Liu S, Li FJ, Taniguchi O and Kawano MM: Requirements of src family kinase activity associated with CD45 for myeloma cell proliferation by interleukin-6. Blood 99: 2172-2181, 2002

28. Leonard WJ: Role of Jak kinases and STATs in cytokine signal transduction. Int J Hematol 73: 271-277, 2001.

29. Brown R, Yang S, Weatherburn C, Gibson J, Ho PJ, Suen H, Hart D and Joshua D: Phospho-flow detection of constitutive and cytokine-induced pSTAT3/5, pAKT and pERK expression highlights novel prognostic biomarkers for patients with multiple myeloma. Leukemia 29: 483-490, 2015.

30. Hundemer M, Klein U, Hose D, Raab MS, Cremer FW, Jauch A, Benner A, Heiss C, Moos M, Ho AD and Goldschmidt H: Lack of CD56 expression on myeloma cells is not a marker for poor prognosis in patients treated by high-dose chemotherapy and is associated with translocation $\mathrm{t}(11 ; 14)$. Bone Marrow Transplant 40: 1033-1037, 2007. 